\title{
Tardive dyskinesia in schizophrenia ${ }^{\dagger}$
}

Peter F. Liddle

\section{Summary}

Sarró et al report grey matter deficits associated with tardive dyskinesia in schizophrenia. Much evidence suggests that the intrinsic pathophysiology of schizophrenia contributes to predisposition to tardive dyskinesia. The possibility that antipsychotics might play a causal role in the grey matter deficits cannot be excluded, but the evidence is tenuous.

\section{Declaration of interest}

In the past 3 years, P.F.L. has received an honorarium for chairing an educational meeting sponsored by Bristol-Myers Squibb.
Peter F. Liddle is Professor of Translational Neuroimaging at the Institute of Mental Health, University of Nottingham. He is a psychiatrist and neuroscientist who employs structural and functional imaging techniques to investigate the mechanism of the symptoms of schizophrenia.

Sarró et al $^{1}$ report that in patients with schizophrenia, tardive dyskinesia is associated with widespread deficits in the amount of cerebral grey matter, most prominently in the basal ganglia and thalamus, but also in frontal and temporal cortex. The word 'tardive', denoting delayed onset following initiation of antipsychotic treatment, implies that antipsychotic medication plays a substantial causal role in tardive dyskinesia. However, recurring dyskinetic movements of facial and limb muscles, indistinguishable in form from tardive dyskinesia, are observed in patients with schizophrenia who have never been exposed to antipsychotics. $^{2}$ Therefore, understanding the pathophysiology of dyskinesia in schizophrenia assumes special clinical importance. Evidence of association observed in cross-sectional imaging studies cannot prove cause, but relevant experimental investigations would be unethical and impractical in humans. This means that we must endeavour to draw what conclusions we can from the associations revealed by cross-sectional imaging studies.

\section{Comparison with auditory hallucinations}

Because brain imaging has been employed more extensively in the investigation of other symptoms of schizophrenia, it is instructive to compare what imaging has revealed about dyskinesia with what has been learned about auditory hallucinations. Like dyskinesia, hallucinations are transient phenomena typically lasting a matter of seconds, but nonetheless usually recurring on multiple occasions in schizophrenia. This time course implies that symptom manifestation is likely to reflect transient aberrant neurotransmission in the relevant brain circuit occurring against a background of a persistent predisposing factor.

Dopamine blockade with antipsychotics can suppress both hallucinations and dyskinesia, at least in the short term, ${ }^{3}$ suggesting that the expression of both types of symptom involves transient excessive dopaminergic transmission. Strong evidence from imaging studies indicates that an excess of dopamine in presynaptic terminals in the corpus striatum plays a cardinal role in the pathophysiology of schizophrenia, ${ }^{4}$ most markedly during the acute phase of illness when hallucinations are prominent.

'See pp. 51-57, this issue
However, no evidence of a specific association between indices of increased dopaminergic transmission and tardive dyskinesia has yet been established.

With regard to the nature of predisposing factors, metaanalysis provides clear evidence that auditory hallucinations in schizophrenia are associated with a deficit of grey matter in a network of brain regions, including superior temporal gyrus and insula. $^{6}$ In contrast, the deficits associated with dyskinesia reported by Sarró et al ${ }^{1}$ were more prominent in basal ganglia and dorsomedial thalamus, but were also discernible in frontal and temporal cortex. Thus, in both dyskinesia and hallucinations, deficits in grey matter are relatively widespread, and embrace brain regions that would be expected to be engaged during expression of the relevant symptoms: basal ganglia in the case of dyskinesia and superior temporal gyrus in the case of auditory hallucinations. It is noteworthy that in the case of dyskinesia, the implicated region of the basal ganglia is the caudate nucleus rather than putamen. Although the putamen is engaged in execution of simple motor actions, the caudate is part of a circuit including dorsomedial thalamus and frontal cortex that is engaged in executive control of motor actions. Similarly, in the case of auditory hallucinations, the implicated regions include not only the auditory processing regions in superior temporal gyrus, but also the insula which plays a key role in the allocation of attention to stimuli according to their behavioural salience. ${ }^{7}$

\section{The pathophysiology of schizophrenia}

Despite extensive investigation, many details of the pathophysiology of schizophrenia remain uncertain. Nonetheless, there is compelling evidence that a pathological process affecting microstructural elements of brain cells leads to subtle deficits in the amount of grey matter. In most cases the amount of grey matter lies within the normal range, and the deficit is only discernible by examining group averages. The nature of the microstructural abnormality remains uncertain, but much evidence is consistent with a disorder of synaptic structure that results in aberrant communication between brain regions. ${ }^{8}$ Symptoms arising from this putative deficit in neural communication are likely to depend on which brain regions are affected by the microstructural pathology. Furthermore, this communication deficit is apparently exacerbated by imbalances in dopaminergic neurotransmission. Disordered dopaminergic transmission might arise from exogenous factors such as drugs, or from endogenous factors, including the possibility that the microstructural pathology intrinsic to the illness might act directly on brain regions that regulate dopaminergic transmission. 
Perhaps the most parsimonious interpretation of the evidence is that the major predisposing factor underlying all the symptoms characteristic of schizophrenia, including hallucinations and dyskinesia, is a similar microstructural abnormality that affects communication between brain regions, with the form of symptoms being determined by which brain regions are involved. However, before concluding that it is likely that a similar microstructural pathology contributes to hallucinations and dyskinesia, it is important to consider respects in which hallucinations and dyskinesia differ.

\section{Other predisposing factors}

First, the two types of symptoms differ in age dependence. The prevalence of orofacial dyskinesia in schizophrenia increases markedly with age, suggesting that age-related degeneration plays a significant role in many cases. ${ }^{9}$ Of relevance to the relationship between the pathophysiology of schizophrenia and dyskinesia, patients with more severe negative symptoms tend to have onset of dyskinesia at a younger age. However, even in cases with substantial negative symptoms, the mean age at onset is in the mid-40s, ${ }^{9}$ whereas onset of the florid psychotic symptoms of schizophrenia, including hallucinations, typically occurs two decades earlier. Although the occurrence of hallucinations in dementia suggests that age-related degeneration can contribute to hallucinations, the association with aging is much stronger in the case of dyskinesia. Age-related degeneration might account for the observed tardy onset of dyskinesia.

Isolated hallucinations and dyskinesia can both occur in otherwise healthy individuals, but the predisposing factors tend to differ. For example, whereas ageing increases the likelihood of dyskinesia, sleep deprivation is associated with hallucinations. Despite the pharmacological similarities, such as acute suppression of both hallucinations and dyskinesia by dopamine blockers, ${ }^{3}$ there are differences. There is relatively little overlap between drugs considered to be hallucinogenic and those that are reported to cause dyskinesia. Most important is the observation from naturalistic clinical studies that treatment with a higher dose of antipsychotics is associated with a greater risk of dyskinesia, ${ }^{10}$ although the likelihood that more severe cases are prescribed higher doses should be borne in mind. Cautious reduction in dose is often recommended for the management of tardive dyskinesia, although there is little evidence to support this recommendation. Despite the lack of evidence from appropriately controlled studies, a substantial body of evidence from observation of routine clinical practice does suggest that antipsychotic drugs play a causal role in tardive dyskinesia. ${ }^{11}$ Overall, the evidence indicates that it would be far too simplistic to conclude that the difference in the pathophysiology of hallucinations and of dyskinesia is merely a matter of differences in the brain regions affected by the pathological process intrinsic to schizophrenia.

From the clinical perspective, a critical issue is whether or not the deficits in grey matter reported by Sarró et al ${ }^{1}$ might actually be caused by prolonged exposure to antipsychotic drugs. Whether macroscopic changes in brain structure are attributable to antipsychotics is unclear. Both increases and decreases in volume of the basal ganglia have been reported. ${ }^{12}$ There have been thought-provoking reports of widespread decreases in cortical grey matter in schizophrenia during long-term treatment with antipsychotic medication, ${ }^{13}$ but disentangling effects of greater severity of illness from the direct effects of medication is challenging. Thus, at this stage, the grounds for proposing that the deficit of grey matter associated with tardive dyskinesia might be a direct consequence of antipsychotic treatment are tenuous.

\section{Conclusion}

In conclusion, the evidence that recurrent dyskinesia is a manifestation of schizophrenia, together with the compelling evidence that the intrinsic pathophysiology of schizophrenia is associated with widespread but subtle deficits in the amount of grey matter, supports the hypothesis that the intrinsic pathophysiological process of schizophrenia, acting in brain regions that are engaged in complex motor activity, makes a substantial contribution to the predisposition to tardive dyskinesia. However, comparison of dyskinesia with hallucinations indicates that factors other than the brain regions affected by the intrinsic pathophysiology play a role in the determining predisposition to these two disparate types of symptoms. In particular, ageing plays a much greater role in dyskinesia and might be a key factor in the observed tardiness. The possibility that treatment with antipsychotic medication might play a direct causal role in the deficit of grey matter reported by Sarró et al ${ }^{1}$ cannot be excluded, but at this stage the evidence for such a role is tenuous.

Peter F. Liddle, BMBCh, PhD, MRCPsych, University of Nottingham, Institute of Mental Health, Triumph Road, Nottingham NG6 2TU, UK. Email: peter.liddle@nottingham.ac.uk

First received 28 Jan 2013, final revision 14 May 2013, accept 14 May 2013

\section{References}

1 Sarró S, Pomarol-Clotet E, Canales-Rodríguez EJ, Salvador R, Gomar JJ, Ortiz-Gil J, et al. Structural brain changes associated with tardive dyskinesia in schizophrenia. Br J Psychiatry 2013; 203: 51-7.

2 Owens DG, Johnstone EC, Frith CD. Spontaneous involuntary disorders of movement: their prevalence, severity, and distribution in chronic schizophrenics with and without treatment with neuroleptics. Arch Gen Psychiatry 1982; 39: 452-61.

3 Glazer WM, Hafez H. A comparison of masking effects of haloperidol versus molindone in tardive dyskinesia. Schizophr Res 1990; 3: 315-20.

4 Kegeles LS, Abi-Dargham A, Frankle WG, Gil R, Cooper TB, Slifstein M, et al. Increased synaptic dopamine function in associative regions of the striatum in schizophrenia. Arch Gen Psychiatry 2010; 67: 231-9.

5 Laruelle M, Abi-Dargham A, Gil R, Kegeles L, Innis R. Increased dopamine transmission in schizophrenia: relationship to illness phases. Biol Psychiatry 1999; 46: 56-72.

6 Palaniyappan L, Balain V, Radua J, Liddle PF. Structural correlates of auditory hallucinations in schizophrenia: a meta-analysis. Schizophr Res 2012; 46: 1249-56.

7 Palaniyappan L, Liddle PF. Does the salience network play a cardinal role in psychosis? An emerging hypothesis of insular dysfunction. J Psychiatry Neurosci 2012; 37: 17-27.

8 Hayashi-Takagi A, Sawa A. Disturbed synaptic connectivity in schizophrenia: convergence of genetic risk factors during neurodevelopment. Brain Res Bull 2010; 83: 140-6.

9 Liddle PF, Barnes TR, Speller J, Kibel D. Negative symptoms as a risk factor for tardive dyskinesia in schizophrenia. Br J Psychiatry 1993; 163: 776-80.

10 Morgenstern $\mathrm{H}$, Glazer WM. Identifying risk factors for tardive dyskinesia among long-term outpatients maintained with neuroleptic medications. Results of the Yale tardive dyskinesia study. Arch Gen Psychiatry 1993; 50 723-33.

11 Morgenstern H, Glazer WM, Niedzwiecki D, Nourjah P. The impact of neuroleptic medication on tardive dyskinesia: a meta-analysis of published studies. Am J Public Health 1987; 77: 717-24.

12 Ebdrup BH, Nørbak H, Borgwardt S, Glenthøj B. Volumetric changes in the basal ganglia after antipsychotic monotherapy: a systematic review. Curr Med Chem 2013; 20: 438-47

13 Ho BC, Andreasen NC, Ziebell S, Pierson R, Magnotta V. Long-term antipsychotic treatment and brain volumes: a longitudinal study of firstepisode schizophrenia. Arch Gen Psychiatry 2011; 68: 128-37. 